\title{
Cyclone-Anticyclone asymmetry of eddy detection on gridded altimetry product in the Mediterranean Sea
}

A. Stegner ${ }^{1}$, B. Le Vu ${ }^{1}$, F. Dumas ${ }^{2,3}$, M. Ali Ghannami ${ }^{4}$, A. Nicolle ${ }^{4}$, C. Durand $^{5}$, Y. Faugere ${ }^{5}$

\author{
${ }^{1}$ LMD, CNRS-IPSL, Ecole Polytechnique, Palaiseau, France \\ ${ }^{2}$ SHOM, Research Departement, Brest, France \\ ${ }^{3}$ LOPS, CNRS-IFREMER-IRD-UBO, Plouzané, France \\ ${ }^{4}$ ENSTA-Bretagne, Brest, France
}

Key Points:

- Altimetry

- OSSE

- mesoscale eddies

- Mediterranean sea

Corresponding author: Alexander Stegner, astegner@lmd.ipsl.fr 


\begin{abstract}
We perform an Observing System Simulation Experiment (OSSE), that simulates the satellite sampling and the mapping procedure on the sea surface of the high-resolution model CROCO-MED60v40, to investigate the reliability and the accuracy of the detected eddies. The main result of this study is a strong cyclone-anticyclone asymmetry of the eddy detection on the altimetry products AVISO/CMEMS in the Mediterranean Sea. Large scale cyclones having a characteristic radius larger than the local deformation radius are much less reliable than large scale anticyclones. We estimate, that less than $60 \%$ of these cyclones detected on gridded altimetry product are reliable, while more than $85 \%$ of mesoscale anticyclones are reliable. Besides, both the barycenter and the size of these mesoscale anticyclones are relatively accurate. This asymmetry comes from the difference of stability between cyclonic and anticyclonic eddies. Large mesoscale cyclones often split into smaller sub mesoscale structures having a rapid dynamical evolution. The numerical model CROCO-MED60v40 shows that this complex dynamic is too fast and too small to be accurately captured by the gridded altimetry products. The spatio-temporal interpolation smoothes out this sub mesoscale dynamics and tends to generate an excessive number of unrealistic mesoscale cyclones in comparison with the reference field. On the other hand, large mesoscale anticyclones, which are more robust and which evolve more slowly, can be accurately tracked by standard altimetry products. We also confirm that the AVISO/CMEMS products induce a bias on the eddy intensity. The azimuthal geostrophic velocities are always underestimated for large mesoscale anticyclones.
\end{abstract}

\title{
Plain Language Summary
}

[ enter your Plain Language Summary here or delete this section]

\section{Introduction}

The increase of the spatial resolution in both numerical models and remote sensing observations (altimetry and visible image) revealed the prevalence of mesoscale eddies throughout the oceans. These coherent structures can survive several months or even years (Puillat et al., 2002; Ioannou et al., 2017; Nencioli et al., 2018; Laxenaire et al., 2019). They are able to trap and transport heat, mass and biogeochemicals properties from their regions of formation to remote areas. For instance, the mean trajectories of the long-lived Agulhas Rings control the global transport in the Southern Ocean (Dencausse et al., 2010; Laxenaire et al., 2018). In the Mediterranean Sea, the trajectories and the merging or splitting of the long-lived Algerian eddies have an impact on the regional transport of Atlantic Water and Levantine Intermediate Water in the Algerian Basin (Escudier et al., 2016; Garreau et al., 2018). Moreover, these long-lived mesoscale eddies can have a strong impact on biological productivity and on the upper-ocean ecology and biogeochemical cycles (Mcgillicuddy et al., 1998; d'Ovidio et al., 2010; Lévy et al., 2018; Cotroneo et al., 2016) especially in an oligotrophic area. Hence, the movement of pelagic species can be strongly influenced by the dynamics of mesoscale eddies and their surrounding filaments (Cotté et al., 2011; Abrahms et al., 2018; Baudena et al., 2019) and some fisheries track lagrangian coherent structures associated to eddies (Prants et al., 2012; Budyansky et al., 2017; Watson et al., 2018; Arur et al., 2020). Therefore, the dynamics of mesoscale eddies have a significant impact on the surface circulation and oceanic biogeochemistry at both local and regional scales.

However, a correct assessment of the dynamical characteristics of real mesoscale eddies is still a challenge. On one hand, direct in-situ observations provided by oceanographic campaigns or autonomous platforms (Argo profilers or gliders) can be very accurate but they remain sparse. On the other hand, remote sensing observations cover almost all oceans and provide every day a large amount of data to detect a very large number of eddies. These detections are mainly derived by analyzing satellite altimetry 
gridded fields which provide daily maps of sea surface height and surface geostrophic velocity that are not affected by cloud coverage. These gridded altimetry products, are used by all the eddy detection algorithms, that have been developed these last ten years, to identify automatically mesoscale eddies at the ocean surface (Chaigneau et al., 2009; Chelton et al., 2007; Doglioli et al., 2007; Nencioli et al., 2010; Chelton et al., 2011; Mason et al., 2014; Le Vu et al., 2018). This methodology, which makes it possible to locate the center of the eddies and estimate their size and intensity, is widely used in oceanography and has led to a very large number of research papers in recent years. But, surprisingly, very few works have studied the reliability and the accuracy of the eddy characteristics derived from standard altimetry products.

Few studies performed quantitative comparisons of the eddy sizes and intensities between the estimations derived from altimetry products and those measured directly by surface buoys trajectories or VMADCP (Mkhinini et al., 2014; Ioannou et al., 2017; Garreau et al., 2018; Ioannou et al., 2019). The number of these studies is necessarily limited by the number of in-situ observations that have been carried out in the core of oceanic eddies. However, all of them show, for various Mediterranean anticyclones, a systematic underestimation of the AVISO/CMEMS surface geostrophic velocity in comparison with in-situ velocity measurements. Even if mesoscale eddies are generally considered to be geostrophic, for few intense anticyclones the ageostrophic velocity components induced by the local curvature of the streamlines are not negligible (Penven et al., 2014; Douglass \& Richman, 2015; Ioannou et al., 2019). The iterative scheme proposed by Ioannou et al. (2019), to compute the cyclostrophic velocity components, shows for one intense anticyclone that the corrected velocity fields were much closer to the in-situ observations. However, the wide majority of mesoscale eddies are not concerned by this specific correction and the systematic underestimation of the eddy intensity might be due to the spatiotemporal heterogeneity of the tracks of altimetry satellites rather than the geostrophic assumption.

Recently, the pioneering work of Amores et al. (2018) performed several Observing System Simulation Experiment (OSSE) to simulate the along-track satellite measuring process and generate satellite like SLA gridded maps for the North Atlantic Ocean and the Mediterranean Sea. They were therefore able to investigate how the dynamical properties of the detected eddies are influenced by the satellite sampling and the mapping procedure. They emphasize on the spatial resolution of the gridded altimetry product which is not enough to capture the small eddies that are the most abundant in the high-resolution simulations used as a ground truth. According to this analysis, we could detect on the AVISO/CMEMS products only $16 \%$ of the total number of eddies in the Mediterranean Sea. The unresolved structures are aliased into larger structures and therefore the gridded altimetry products contain an unrealistic number of large mesoscale eddies. However, this study performed a global statistical analysis without any distinction between cyclonic and anticyclonic eddies while the analysis of Chelton et al. (2011) in the global ocean have shown some statistical evidences that large mesoscale anticyclones live, on average, longer than their cyclonic counterparts. This cyclone-anticyclone asymmetry, in the lifetime of mesoscale eddies is even more pronounced in the Mediterranean Sea (Mkhinini et al., 2014; Stegner et al., 2019; Barboni et al., 2021). Such asymmetry finds an explanation in the dynamical stability and the robustness of mesoscale anticyclones having a characteristic radius larger than the local deformation radius (Arai \& Yamagata, 1994; Stegner \& Dritschel, 2000; Baey \& Carton, n.d.). Moreover, stable anticyclones tend to remain coherent within a turbulent flow (Polvani et al., 1994; Arai \& Yamagata, 1994; Linden et al., 1995) and they were found to be more robust to an external strain or shear than cyclonic eddies (Graves et al., 2006; Perret et al., 2011). Similarly, in the wakes of a large island, where mesoscale eddies of both sign are influenced by the strain of their neighbors, anticyclones are more circular and robust while cyclones, which are larger than the deformation radius, tend to be elongated and distorted (Perret et al., 2006; Dong et al., 2007; Stegner, 2014). In the Mediterannean Sea, where the de- 
formation radius $(\mathrm{Rd}=8-15 \mathrm{~km})$ is small in comparison with the typical radii of mesoscale eddies, both the vortex stability and the vortex-vortex interactions explain the predominance of large-scale anticyclones among the long-lived eddies.

The goal of this paper is to investigate the reliability and the accuracy of mesoscale eddies detected on the AVISO/CMEMS altimetry products in the Mediterranean Sea. Even if a large number of small scale eddies are missed, due to the spatial resolution of the gridded altimetry maps, we will focus on the large-scale eddies that could be accurately detected. How reliable are they ? Are their size and intensity correctly quantified ? Aware of the cyclone-anticyclone asymmetry that could affect mesoscale eddies, we will perform this analysis separately for cyclones and anticyclones. Moreover, we will also compare the accuracy of two different types of AVISO/CMEMS products distributed by CMEMS: the delayed time (DT), which takes into account the altimetry tracks forward and backward in time, and the near real time (NRT) which considers only the past tracks.

As Amores et al. (2018) we performed an OSSE of the AVISO/CMEMS products using the SSH of a numerical simulation having a much higher spatial resolution than the altimetry products. This numerical simulation of the regional circulation of the Mediterranean Sea was performed with the CROCO model with at $1 / 60^{\circ}$ and was used as the ground truth for the OSSE. Then the automatic eddy detection algorithm AMEDA (Le Vu et al., 2018) was used to detect and quantify the dynamical characteristics of the mesoscale eddies both on the numerical model and the simulations of the DT and NRT AVISO/CMEMS products. The paper is organized as follows. We first present in section 2 the CROCO ocean model used for our realistic numerical simulations of the Mediterranean Sea in 2015 and 2016. The mean features of the general circulation are compared with the observations to check the consistency of the run CROCO-MED60v40-15-16. The methodology used for the Observing System Simulation Experiment (OSSE) and the eddy detection algorithm are presented in section 3 . The main results on the cyclone-anticyclone asymmetry are detailed in section 4 . Then, we discuss, in section 5 , the dynamical origin of the asymmetry and finally we conclude in section 6 .

\section{High-resolution model of the Mediteranean Sea}

We used the results of a realistic numerical simulation that was carried out for the Mediterranean Sea using the CROCO numerical model (http://www.croco-ocean.org). For more details on the numerical characteristics of the CROCO model we refer to Shchepetkin and McWilliams (2005), Debreu et al. (2012), and Auclair et al. (2018). The simulation under investigation, CROCO-MED60v40-15-16, was forced at the ocean surface with ARPEGE HR analysed meteorological fields (winds, pressure, air temperature, relative humidity) thanks to the classical bulk COARE formula (Fairall et al., 2003). The standard primitive equations are solved with an horizontal resolution of $1 / 60^{\circ}$ in both longitudinal and latitudinal directions. The vertical coordinate used is a generalised terrain following one. It is stretched to keep as flat as possible the levels near the surface whatever the bathymetry gradient is. Fourty unevenly distributed vertical levels discretized the water column. They are closer one from each other next to the surface and more spaced by the bottom where the vertical gradients of hydrology parameters (temperature or salinity) are weak. The initial and the boundary conditions were built from CMEMS global system analysis optimally interpolated on the computational grid. CROCO-MED60v40-15-16 is a result of a free run simulation (no nudging nor assimilation of any kind) that started on the 1st of August 2012 when the water column stability is at its maximum to avoid static instability in the spinning up phase. It ran till the end of December 2016.

To test the realism of the regional surface circulation of this numerical model, we compute the mean geostrophic eddy kinetic energy MKEg and compare it with the AVISO/CMEMS observations during the 2012-2016 period. Following the work of Pujol and Larnicol (2005), 
we extract the low frequency (LF) signal applying a Butterworth low-pass time filter of order 4 to the geostrophic velocity field, derived from the SSH, with a cut-off frequency of 60 days. We have checked that our comparison is weakly sensitive to the cut-off frequency of the low-pass filter (30, 60 or 100 days). According to the figure 2, this MKEg range between $40 \mathrm{~cm}^{2} . \mathrm{s}^{2}$ in the Gabes Gulf to $10^{4} \mathrm{~cm}^{2} . \mathrm{s}^{2}$ in the Alboran Sea. Both the CROCO-MED60v40 model and the AVISO/CMEMS observations exhibit strong MKEg values along the Algerian coast, in the Ionian and the Levantine basins. Most of the regional patterns of MKE are similar in the model and the observation. However, due to the high spatial resolution of the numerical model, small scale patterns such as the North Ligurian current and the Rhodes Gyre current appear to be much stronger in CROCOMED60v40. Besides this statistical validation against altimetric data Ioannou et al. (2020) showed that the numerical solution CROCO-MED60v40-15-16 exhibits realistic mesoscale features even after 3 years of free simulation : some of these features are even very commensurable with observations (density anomaly, main dynamical parameters and tracking of a mesoscale eddy). More generally the simulated hydrology has been systematically compared to the CORA v5.2 dataset and to SST fields from Meteosat SEVIRI imager. These comparisons give reasonable errors for a free run :

- lower $2^{\circ} \mathrm{C}$ everywhere for the SST field,

- ower than $1^{\circ} \mathrm{C}$ and $0.25 \mathrm{psu}$ within the first $200 \mathrm{~m}$,

- lower than $0.5^{\circ} \mathrm{C}$ and 0.1 psu between $200 \mathrm{~m}$ and $600 \mathrm{~m}$,

- less than $0.1^{\circ} \mathrm{C}$ and 0.02 psu below

For the purposes of this paper, we extracted all dynamical fields and especially the daily mean SSH field and we focused our analysis on the last two years: 2015 and 2016.

\section{Methods}

\subsection{OSSE principles}

In order to quantify the reliability and the accuracy of mesoscale eddies detected on the AVISO/CMEMS altimetry products in the Mediterranean Sea, an Observing System Simulation Experiment (OSSE) is performed in a four-satellite configuration, composed of the reference mission Jason-3 and three other missions Sentinel3-A, Sentinel3B, and Cryosat-2. As the CROCO-MED60v40-15-16 resolves the response of the ocean to atmospheric pressure disturbances, it contains large-scale high-frequency signals that cannot be handled by the mapping method based on Optimal Interpolation. Thus, as in the operational processing, a Dynamic Atmospheric Correction (DAC) derived from atmospheric forcing fields (Carrère \& Lyard, 2003) is used to correct this effect. The SWOT simulator software (Gaultier et al., 01 Jan. 2016) is then used to generated along track with realistic measurement errors and noise. The resulting ground truth references are finally ingested in the AVISO/CMEMS mapping procedure (Taburet et al., 2019) to compute gridded fields.

The OSSE SSH (figure 1) corresponds to the $1 / 8^{\circ}$ ADT maps which are identical to the Mediterranean Sea gridded L4 sea surface heights reprocessed available on Corpernicus Marine Environment Monitoring Service (CMEMS). In order to compare the accuracy of the delayed time (DT) and the near real time (NRT) products, two distinct OSSE were performed. For the OSSE-SSH-DT maps the optimal interpolation of individual altimetry tracks (Le Traon et al., 1998) is made on backward and forward tracks within a time window of $+/-5$ days. On the other hand, the OSSE-SSH-NRT maps are built every day with the altimetry tracks of the past ten days. In a second step we derived from the daily mean SSH of CROCO-MED60v40-15-16 the geostrophic velocity field ( $\mathrm{Ug}, \mathrm{Vg}$ ) using the 9-stencil method of Arbic et al. (2012) which is used in the operational processing chain of the AVISO/CMEMS products to derived surface velocity fields. 


\subsection{AMEDA algorithm}

We apply the automatic eddy detection algorithm AMEDA (Le Vu et al., 2018) on the surface geostrophic velocities of the two OSSE and the CROCO-MED60v40-1516 to compare quantitatively the dynamical characteristics of the detected oceanic eddies. The eddy centers which correspond to an extremum of the local normalized angular momentum are first identified. The streamlines surrounding this center are then computed (figure 3a). The mean radius $\langle R\rangle$ and the mean velocity $\langle V\rangle$ are then computed along each closed streamline. This mean radius $\langle R\rangle$ is defined as the equivalent radius of a disc with the same area $\mathrm{A}$ as the one delimited by the closed streamline (equation (1)), while the mean velocity amplitude $\langle V\rangle$ is derived from the circulation along the closed streamline $\mathrm{C}$, where $L_{p}$ is the streamline perimeter (equation(2)).

$$
\begin{gathered}
\langle R\rangle=\sqrt{\left(\frac{A}{\pi}\right)} \\
\langle V\rangle=\frac{1}{L p} \oint V d l
\end{gathered}
$$

We plot in figure 3b the pair of mean eddy velocity $\langle V\rangle$ and mean radius $\langle R\rangle$ for each closed streamlines of a mesoscale anticyclone located in the center of the Ionian Sea. We can see on this example that the mean velocity increases when the radius increases until a maximum velocity $V_{\max }$ is reached. This characteristic radius is labeled $R_{\max }$, and also called the speed radius (Chelton et al., 2011; Laxenaire et al., 2018; Le Vu et al., 2018). This eddy radius $R_{\max }$ is used to quantify the eddy size. The characteristic contour of the detected eddy (the blue thick contour in figure 3a) is associated with the closed streamline of maximal speed. The velocity $V_{\max }$ is used to quantify the eddy intensity. Once this maxima is reached, the azimuthal speed of the eddy decreases until the last closed streamline where $\langle R\rangle=R_{\text {end }}$. The latter is plotted with a blue dashed line in figure 3a. The eddy shape is characterized by two geometrical parameters. The first one is the ellipticity of the closest ellipse that fits the characteristic contour. The second one is the steepness parameter which is used to fit the mean velocity profile $\langle V\rangle$ $=\mathrm{F}(\langle R\rangle)$, of quasi-circular eddies (ellipticity $<0.2$ ), with a generic function:

$$
V_{\theta}(r)=\frac{V_{\max }}{R_{\max }} r e^{\left(1-\left(r / R_{\max }\right)^{\alpha}\right) / \alpha}
$$

where $\alpha$ is the steepness parameter. Such generic profiles were used by (Carton et al., 1989; Stegner \& Dritschel, 2000; Lazar et al., 2013; Yim et al., 2019) to study the stability of various isolated eddies. Note, that when $\alpha=2$, the velocity profile corresponds to a Gaussian vortex, while in the example shown in figure 3b, the steepness parameter is about $\alpha=2.9$.

To quantify the eddy amplitude $\eta_{e d d y}$ we compute the difference between the SSH at the eddy center, where the free surface deviation is maximal, and the mean SSH along the last closed contour (figure 3c).

\subsection{DYNED-Atlas data base}

In order to compare the statistical properties of the eddies detected in the OSSEDT and OSSE-NRT with real eddies detected on the standard AVISO/CMEMS product, we also used the dynamical eddy data-base DYNED-Atlas (https://www.lmd.polytechnique.fr/dyned/). This recent data-base provides 18 years (2000-2018) of eddy detection and tracking in the Mediterranean Sea along with the co-localisation of Argo floats for each detected eddy (https://doi.org/10.14768/2019130201.2). The dynamical characteristics of the eddies 
contained in the DYNED-Atlas database were computed with the AMEDA eddy detection and tracking algorithm applied on mean daily surface velocity fields which include the ageostrophic corrections proposed by Ioannou et al. (2019). We will then build climatological histograms of the main dynamical parameters $\left(R_{\max }, V_{\max }, \eta_{\text {eddy }}\right)$ of the detected eddies during this 18 year period in the Mediterranean Sea.

\section{Results}

\subsection{Statistical analysis reveals the cyclone-anticyclone asymmetry}

We first present, in figure $4 \mathrm{a}$, the histograms of the characteristic eddy radius $\left(R_{\max }\right)$ and the eddy amplitude $\eta_{e d d y}$ for all the detected eddies, regardless of their sign, in the geostrophic surface velocity field of CROCO-MED60v40-15-16 (black curve), the OSSEDT (pink solid curve) and the OSSE-NRT (pink dashed curve). We also add the histogram of the DYNED-Atlas data base (green curve), which corresponds to the eddy detection performed on the AVISO/CMEMS altimetry products, for the same two years 2015-2016. In Figure $4 \mathrm{~b}$, the histograms of the eddy amplitude are plotted for the same four datasets. As Amores et al. (2018), we found that a large fraction of the eddy spectrum are not detected on the standard altimetry products, both the OSSE's and the real AVISO/CMEMS. It makes sense that the $1 / 8^{\circ}$ coarse-resolution products cannot capture the submeso scale eddies $\left(R_{\max }<10 \mathrm{~km}\right)$, which are resolved in the high-resolution CROCO-MED model at $1 / 60^{\circ}$. However, it is more surprising to find that the number of larger eddies $\left(R_{\max }>\right.$ $24 \mathrm{~km}$ ), with radius large enough to be resolved by gridded altimetry products, is systematically overestimated. Amores et al. (2018) found the same behavior, that small eddies are seen by the coarse-resolution altimetry products as bigger structures, and they called such phenomenon the coarsening artefact. However, they did not explain the physical origin of this artefact even for very large eddies that should be accurately resolved by the AVISO/CMEMS products. We should mention here that the characteristic eddy radius used by Amores et al. (2018) is based on the last closed contour and therefore leads to larger values than the characteristic eddy radius $R_{\max }$ we used. In addition to this coarsening artefact a systematic underestimation of the eddy amplitude is also found in Figure 4b which is similar to the figure 5(f) of Amores et al. (2018).

However, if we plot the same histogram but separately for the anticyclonic and the cyclonic eddies we get a very different picture. The size histogram of large-scale anticyclonic eddies coincide between the reference and the OSSE (figure 5a) while the overestimation of large scale eddies is strongly amplified for cyclonic vortices (Figure 5b). The coarsening artefact, depicted by Amores et al. (2018), only concerns cyclonic eddies. Hence, a strong cyclone-anticyclone asymmetry appears for the detection of eddies on gridded altimetry products in the Mediterranean Sea. Taking into account only the statistical aspect, large anticyclones seem to be relatively well captured by the standard AVISO/CMEMS products and correctly detected by the AMEDA algorithm.

In order to filter out small-scale eddies which, obviously, cannot be properly captured by coarse resolution products, we will focus a large part of our analysis to oceanic eddies having a characteristic radius larger than $R_{\max }>24 \mathrm{~km}$. The statistical distributions of the size and the intensity of these mesoscale eddies are depicted in figure 6 . By construction the size histogram of the reference coincide with anticyclonic eddies of the OSSE (figure 6a) while the number of mesoscale cyclones detected is strongly overestimated (by a factor 4 or 5 ) both in the OSSE and the AVISO/CMEMS product in comparison with the reference model CROCO-MED60v40-15-16. On the other hand, the number of intense mesocale eddies, having for instance an azimuthal geostrophic velocity that exceeds $V_{\max }>25 \mathrm{~cm} / \mathrm{s}$, is systematically underestimated in the OSSE and the AVISO/CMEMS. This statistical bias concerns both cyclones and anticyclones and corresponds to an underestimation of the SSH gradients which is probably due to the smoothing induced by the spatio-temporal interpolation of the altimetry tracks. 
Such asymmetry in the detection of mesoscale eddies on altimetry products calls into question all the statistical analysis performed so far in the Mediterranean Sea. For instance, the study of Mkhinini et al. (2014) has shown that large eddies in eastern basin which live more than 21 weeks were predominantly anticyclonic, while Chelton et al. (2011) have shown that the dominance of anticyclones in world ocean occurs only when their lifetime exceeds 45 weeks. We plot in figure 7 the cumulative histogram of the ratio cyclone/anticyclone as a function of the eddy lifetime for the various data sets. When we consider all the eddy sizes (figure 7a) for the whole Mediterranean Sea, the detection performed on the standard AVISO/CMEMS products shows that the predominance of anticyclones occurs when the lifetime exceeds 50 weeks. However, this threshold is reduced when the automatic eddy detection is performed on the OSSE data-set (20-25 weeks) or on the reference model CROCO-MED60v40-15-16 (15 weeks). If now we focus the analysis on mesoscale eddies (i.e. $R_{\max }>24 \mathrm{~km}$ ) anticyclones are predominant whatever their lifetimes in the reference model while in the different OSSE we found a threshold that varies between 18 to 25 weeks (figure $7 \mathrm{~b}$ ). Hence, the predominance of long-lived anticyclones in the Mediterranean Sea, and probably in the world ocean, appears to be much more pronounced than the previous estimations made on standard altimetry products.

\subsection{Reliable/unreliable eddy detection}

A global statistical analysis gives a first estimate of the level of error but does not allow to know precisely, among the detected vortices, the percentage of reliable and erroneous eddy detection. In order to quantify the reliability of the eddy detection, we have, for each vortex detected in the OSSE maps, tried to identify the corresponding vortex in the reference field CROCO-MED60v40-15-16. Therefore, we are looking, if there is one (or more) vortex in the reference field which is inside the characteristic contour calculated in the OSSE field. The series of images presented in figure 8 illustrates the three possible cases that may occur. If the vortex detected in the OSSE field is superimposed on a single vortex in the reference field (i.e. cyclones in figure $8 \mathrm{~b}$ and $8 \mathrm{~h}$ ) it is called "single" and corresponds to a reliable detection. If the vortex detected in the OSSE field is superimposed on several vortices in the reference field, for instance, the cyclone in figure $8 \mathrm{f}$ is superimposed on five cyclones in figure $8 \mathrm{e}$, it is called "multiple". If the vortex detected in the OSSE field is not superimposed on any vortex in the reference field (i.e. figure $8 \mathrm{c}$ and $8 \mathrm{~d}$ ) it corresponds to a "ghost" eddy. In what follows "ghost" and "multiple" eddy detection will be considered as not reliable. The snapshots of figure 8 depicts the temporal evolution of a dipolar structure detected on the OSSE-DT fields in the middle of the eastern basin from December 2015 to March 2016. We can see, for this specific case, that the detection of the mesoscale anticyclone is reliable during the whole period while the detection of the large cyclonic structure, attached to this anticyclone, is often unreliable and varies from "single" to "ghost" or "multiple" detection.

We plot in figure 9 the percentage of ghosts, multiples and single among the detected eddies as a function of the characteristic radius both for anticyclones and cyclones. As expected, the percentage of "ghost eddy" decreases when their size increases. For small eddies, which have a radius smaller or equal to $R_{\max }=15 \mathrm{~km}$ the percentage of "ghosts" exceeds 65 . While for mesoscale eddies having a radius larger than $R_{\max }=24 \mathrm{~km}$ the percentage of "ghosts" drops below 15 . However, as the vortex size increases, the percentage of multiples in the detection increases, resulting in an overall decrease in reliability for large eddies. This phenomenon is much more pronounced for cyclones which characterize the strong cyclone-anticyclone asymmetry in the reliability of detected mesoscale eddies. The percentage of reliable anticyclones could reach 90 at large scale while it never exceeds 65 for cyclones and drops down to 35 for very large structure. This reliability analysis was made on the OSSE-DT data-set and very similar results were found on the OSSE-NRT fields. 


\subsection{Accuracy of the dynamical parameters of detected eddies}

In addition to knowing whether a vortex detected on altimetry products is reliable (i.e. does it really exist?) it is also important to quantify the accuracy of this detection. We have therefore compared, for each eddy detected on the OSSE-DT data-set, its position, size and intensity compared to the eddy of the reference field CROCO-MED60v4015-16, when it exists. The accuracy of detection according to the radius of each cyclone and anticyclone is plotted separately in figure 10. We first plot the relative error of the center position in figure 10a. On average, the barycenter of the characteristic contour is given with an accuracy that stays below one grid size (i.e. $1 / 8^{\circ}$ ) for mesoscale anticyclones. On the other hand, for cyclones the accuracy is systematically lower and degrades with increasing size. The average distance between the barycenter of detected cyclones and the reference always exceeds one grid size and it could even reach two grid size for very large eddies. A similar cyclone-anticyclone asymmetry occurs on the size of detected eddies. The radius of anticyclonic eddies, detected on the OSSE-DT fields, are systematically more accurate than the cyclonic ones. Indeed, the radius of anticyclones are, on average, overestimated by 20 while the radius of cyclones are overestimated by 50 to 70 .

We have already observed in the global statistical analysis (figure $6(\mathrm{c}, \mathrm{d})$ ), that the intensity of eddies is systematically underestimated on the altimetry OSSE-DT data-sets when their characteristic velocity exceeds $V_{\max }>25 \mathrm{~cm} / \mathrm{s}$. Here we can quantify more precisely this underestimation. In comparison with cyclones, the anticyclones exhibit the strongest errors with a velocity deficit that increases from $20 \%$ to $80 \%$ when the radius decreases (figure 10c). Here again, we confirm that large mesoscale eddies, that are better sampled by the altimetry tracks, are more accurate than smaller ones. However, the anticyclonic asymmetry is reversed and cyclones seem to be more accurate, or at least, less underestimated than anticyclones.

Another specificity of this study is to compare the accuracy of the dynamical parameters of the eddies detected respectively on the DT and the NRT products. The relative errors of the OSSE-NRT are compared with the OSSE-DT in figure 11, for cyclones and anticyclones separately. The delayed time (DT) products, which take into account altimetry tracks both backward and foreward in time are expected to be more accurate than the near real time (NRT) products which consider only past tracks. Surprisingly, such improvement occurs only for anticyclonic eddies. According to figure 11, the level of error on both the position, the size and the intensity of cyclones remains high with no significant differences between the eddies detected on the OSSE-DT and the OSSENRT. It is only for large scale anticyclones that the accuracy of the detection on the OSSEDT is significantly better than on the OSSE-NRT, especially for the eddy position and its intensity.

\section{Discussion}

Both the global statistics and the analysis of the reliability and the accuracy of individual eddy detection reveal that the main sources of errors come either from unresolved sub mesoscale eddies or from large mesoscale cyclones. The dynamical evolution presented in figure 8 (see also the movie in the supplementary material) provides a characteristic illustration of what we have been able to observe visually on multiple occasions by comparing the OSSE and the run CROCO-MED60v40-15-16. Large mesoscale anticyclones tend to be robust and long-lived while various filaments having intense cyclonic vorticity spiral in the periphery. The rapid instability of these filaments generate several submesoscale cyclones that evolve very rapidly between large anticyclones. The fast dynamics of these small cyclonic structures cannot be tracked or properly sampled by the altimetry tracks. Hence, the spatio-temporal interpolation (Le Traon et al., 1998), used to build the standard AVISO/CMEMS products, smooth out this intense submeso 
scale cyclonic activity and generate unrealistic large-scale cyclones on the daily altimetry maps. On the other hand, several studies have shown that large-scale cyclones having a radius that exceed the local deformation radius are more unstable than mesoscale anticyclones (Stegner \& Dritschel, 2000; Baey \& Carton, n.d.). Moreover, in a turbulent environment and submitted to the external strain of neighbouring eddies, large cyclones are easily distorted into elliptical structures and often split into smaller eddies (Arai \& Yamagata, 1994; Graves et al., 2006; Perret et al., 2011). Hence, even if a large scale cyclone is formed, among the turbulent oceanic eddy field, its longevity and robustness will be reduced due to its dynamical stability properties. Therefore, the coarsening artifact, revealed by Amores et al. (2018), applies mainly to cyclonic eddies. This artifact finds its explanation in the dynamical properties of the turbulent eddy field which tend to favor the formation of long-lived anticyclones. The result is that large mesoscale anticyclones, which are more robust and that evolve more slowly, can be spatially resolved and accurately tracked by standard altimetry products.

\section{Conclusion}

Thanks to a Observing System Simulation Experiment (OSSE) that simulate the along-track satellite measuring process on the sea surface of the high resolution model CROCO-MED60v40-15-16 we investigate how the reliability and the accuracy of the detected eddies are influenced by the satellite sampling and the mapping procedure. The main result of this study is that there is a large difference in reliability between the detection of cyclonic and anticyclonic mesoscale eddies on the gridded altimetry products AVISO/CMEMS of the Mediterranean Sea distributed by CMEMS. This asymmetry comes from the difference of stability between large scale cyclones and anticyclones. Mesoscale anticyclones having a characteristic radius that exceed the deformation radius tend to be more stable or robust to external strain than cyclones having equivalent size. It implies that large mesoscale cyclones often splits into smaller sub mesoscale structures having a rapid dynamical evolution. This complex dynamic is too fast and too small to be accurately captured by the gridded altimetry products based on a strong spatio-temporal interpolation (Le Traon et al., 1998). The later smooth out this sub mesoscale dynamics and tend to generate an excessive number of unrealistic (i.e. unreliable) mesoscale cyclones in comparison with the reference field. We found that the both the reliability and the accuracy of the detected cyclones decrease when their characteristic radius $R_{\max }$ are larger than $35 \mathrm{~km}$. We estimate, in the Mediterranean Sea, that less than $60 \%$ of the mesoscale cyclones detected on gridded altimetry product are indeed reliable.

On the other hand, we found that the reliability and the accuracy of large scale anticyclones increase when their size increase. We estimate, that more than $85 \%$ of large scale mesoscale anticyclones (i.e. $R_{\max }>2 R_{d}=25 \mathrm{~km}$ ) detected on gridded altimetry product are reliable. Besides, both the position of the center and their size are relatively accurate. The mean error on the location of the eddy barycenter remain below the grid size (i.e. $1 / 8^{\circ}$ ) while the relative error on the characteristic eddy radius do not exceed, on average, $25 \%$. However, we confirm that gridded altimetry products have a systematic bias on the eddy intensity and especially for anticyclones. The maximal azimuthal geostrophic velocity $V_{\max }$ is always underestimated on the AVISO/CMEMS products even for large mesoscale anticyclones.

This study shows the biases that can be induced by the use of gridded altimetry products which are often considered as a reliable observational data-sets for large mesoscale structures. This study shows that AVISO/CMEMS data-sets should be used with caution especially when studying the properties of large cyclonic eddies. One may wonder if other oceanic regions would also be affected by this cyclone-anticyclone asymmetry of eddy detection. The study of Amores et al. (2018) indicates that the coarsening artefact is also significant in the Northern Atlantic even if the local deformation radius is at least two or three times larger than in the Mediterranean Sea. We could then a expect 
a higher accuracy on the detection of mesoscale eddies in the Northern Atlantic. However, the smoothing of the unstable dynamics of large scale cyclones, induced by the optimal interpolation of altimetry tracks, is not only a spatial but also and temporal smoothing. Therefore, a more thorough study that takes into account the global ocean should be considered in the future.

\section{Acknowledgments}

This work and especially Briac Le Vu and Mohammed Ali Ghannami contribution were funded by the Agency Innovation and Defense with the research contract ADTEOC-TR and the French Defence Procurement Agency (DGA) with the research study Protevs 2 and contract Catoobs.

\section{References}

Abrahms, B., Scales, K. L., Hazen, E. L., Bograd, S. J., Schick, R. S., Robinson, P. W., \& Costa, D. P. (2018). Mesoscale activity facilitates energy gain in a top predator. Proceedings of the Royal Society B: Biological Sciences, 285(1885), 20181101. Retrieved from https://royalsocietypublishing .org/doi/abs/10.1098/rspb.2018.1101 doi: 10.1098/rspb.2018.1101

Amores, A., Jordà, G., Arsouze, T., \& Le Sommer, J. (2018). Up to what extent can we characterize ocean eddies using present-day gridded altimetric products? Journal of Geophysical Research: Oceans, 123(10), 7220-7236. Retrieved from https://agupubs.onlinelibrary.wiley.com/doi/abs/ 10.1029/2018JC014140 doi: 10.1029/2018JC014140

Arai, M., \& Yamagata, T. (1994). Asymmetric evolution of eddies in rotating shallow water. Chaos: An Interdisciplinary Journal of Nonlinear Science, 4(2), 163-175. Retrieved from https://doi.org/10.1063/1.166001 doi: $10.1063 / 1.166001$

Arbic, B. K., Scott, R. B., Chelton, D. B., Richman, J. G., \& Shriver, J. F. (2012). Effects of stencil width on surface ocean geostrophic velocity and vorticity estimation from gridded satellite altimeter data. Journal of Geophysical Research: Oceans, 117(C3). Retrieved from https://agupubs.onlinelibrary.wiley .com/doi/abs/10.1029/2011JC007367 doi: 10.1029/2011JC007367

Arur, A., Krishnan, P., Kiruba-Sankar, R., Suryavanshi, A., Kumar, K. L., Kantharajan, G., ... Babu, D. (2020). Feasibility of targeted fishing in mesoscale oceanic eddies: a study from commercial fishing grounds of andaman and nicobar islands, india. International Journal of Remote Sensing, 41(14), 50115045. Retrieved from https://doi.org/10.1080/01431161.2020.1724347 doi: 10.1080/01431161.2020.1724347

Auclair, F., Benshila, R., Debreu, L., Ducousso, N., Dumas, F., Marchesiello, P., \& Lemarié, F. (2018, February). Some Recent Developments around the CROCO Initiative for Complex Regional to Coastal Modeling. In COMOD 2018 - Workshop on Coastal Ocean Modelling (p. 1-47). Hambourg, Germany. Retrieved from https://hal.inria.fr/hal-01947670

Baey, J.-M., \& Carton, X. (n.d.). Vortex multipoles in two-layer rotating shallowwater flows. Journal of Fluid Mechanics, 460, 151-175.

Barboni, A., Lazar, A., Stegner, A., \& Moschos, E. (2021). Lagrangian eddy tracking reveals the eratosthenes anticyclonic attractor in the eastern levantine basin. Ocean Science Discussions, 2021, 1-35. Retrieved from https:// os.copernicus.org/preprints/os-2020-118/ doi: 10.5194/os-2020-118

Baudena, A., Ser-Giacomi, E., d'Onofrio, D., Capet, X., Cotté, C., Cherel, Y., \& d'Ovidio, F. (2019). Fine-scale fronts as hotspots of fish aggregation in the open ocean. bioRxiv. Retrieved from https://www.biorxiv.org/content/ early/2019/12/17/2019.12.16.877571 doi: 10.1101/2019.12.16.877571 
Budyansky, M. V., Prants, S. V., Samko, E. V., \& Uleysky, M. Y. （2017, September). Identification and Lagrangian analysis of oceanographic structures favorable for fishery of neon flying squid (Ommastrephes bartramii) in the South Kuril area. Oceanology, 57(5), 648-660. doi: 10.1134/S0001437017050034

Carrère, L., \& Lyard, F. (2003). Modeling the barotropic response of the global ocean to atmospheric wind and pressure forcing - comparisons with obser-

vations. Geophysical Research Letters, 30(6). Retrieved from https:// agupubs.onlinelibrary.wiley.com/doi/abs/10.1029/2002GL016473 doi: https://doi.org/10.1029/2002GL016473

Carton, X. J., Flierl, G. R., \& Polvani, L. M. (1989). The generation of tripoles from unstable axisymmetric isolated vortex structures. EPL (Europhysics Letters), 9(4), 339. Retrieved from http://stacks.iop.org/0295-5075/9/i=4/a=007

Chaigneau, A., Eldin, G., \& Dewitte, B. (2009). Eddy activity in the four major upwelling systems from satellite altimetry (1992-2007). Progress in Oceanography, 83(1), 117-123.

Chelton, D. B., Schlax, M. G., \& Samelson, R. M. (2011). Global observations of nonlinear mesoscale eddies. Progress in Oceanography, 91(2), 167-216. Retrieved from http://www.sciencedirect.com/science/article/pii/ S0079661111000036 doi: http://dx.doi.org/10.1016/j.pocean.2011.01.002

Chelton, D. B., Schlax, M. G., Samelson, R. M., \& de Szoeke, R. A. (2007). Global observations of large oceanic eddies. Geophysical Research Letters, 34(15). Retrieved from http://dx.doi.org/10.1029/2007GL030812 doi: 10.1029/2007GL030812

Cotroneo, Y., Aulicino, G., Ruiz, S., Pascual, A., Budillon, G., Fusco, G., \& Tintoré, J. (2016). Glider and satellite high resolution monitoring of a mesoscale eddy in the algerian basin: Effects on the mixed layer depth and biochemistry. Journal of Marine Systems, 162(C), 73-88. doi: 10.1016/j.jmarsys.2015.12.004

Cotté, C., d'Ovidio, F., Chaigneau, A., Lévy, M., Taupier-Letage, I., Mate, B., \& Guinet, C. (2011). Scale-dependent interactions of mediterranean whales with marine dynamics. Limnology and Oceanography, 56(1), 219-232. Retrieved from https://aslopubs.onlinelibrary.wiley.com/doi/abs/10.4319/ 1o.2011.56.1.0219 doi: 10.4319/lo.2011.56.1.0219

Debreu, L., Marchesiello, P., Penven, P., \& Cambon, G. ～(2012). Two-way nesting in split-explicit ocean models: Algorithms, implementation and validation. Ocean Modelling, 49-50,1-21. Retrieved from http:// www.sciencedirect.com/science/article/pii/S1463500312000480 doi: http://dx.doi.org/10.1016/j.ocemod.2012.03.003

Dencausse, G., Arhan, M., \& Speich, S. (2010). Routes of agulhas rings in the southeastern cape basin. Deep Sea Research Part I: Oceanographic Research Papers, 57(11), 1406 - 1421. Retrieved from http://www.sciencedirect.com/ science/article/pii/S0967063710001603 doi: https://doi.org/10.1016/ j.dsr.2010.07.008

Doglioli, A. M., Blanke, B., Speich, S., \& Lapeyre, G. (2007). Tracking coherent structures in a regional ocean model with wavelet analysis: Application to cape basin eddies. Journal of Geophysical Research: Oceans, 112(C5). Retrieved from http://dx.doi.org/10.1029/2006JC003952 (C05043) doi: 10.1029/2006JC003952

Dong, C., McWilliams, J. C., \& Shchepetkin, A. F. (2007, 04). Island Wakes in Deep Water. Journal of Physical Oceanography, 37(4), 962-981. Retrieved from https://doi.org/10.1175/JP03047.1 doi: 10.1175/JPO3047.1

Douglass, E. M., \& Richman, J. G. (2015). Analysis of ageostrophy in strong surface eddies in the atlantic ocean. Journal of Geophysical Research: Oceans, 120(3), 1490-1507. Retrieved from https://agupubs.onlinelibrary.wiley.com/ doi/abs/10.1002/2014JC010350 doi: 10.1002/2014JC010350

d'Ovidio, F., De Monte, S., Alvain, S., Dandonneau, Y., \& Lévy, M. (2010). Fluid 
dynamical niches of phytoplankton types. Proceedings of the National Academy of Sciences, 107(43), 18366-18370. Retrieved from https://www.pnas.org/ content/107/43/18366 doi: 10.1073/pnas.1004620107

Escudier, R., Mourre, B., Juza, M., \& Tintoré, J. (2016). Subsurface circulation and mesoscale variability in the algerian subbasin from altimeter-derived eddy trajectories. Journal of Geophysical Research: Oceans, 121(8), 63106322. Retrieved from http://dx.doi.org/10.1002/2016JC011760 doi: 10.1002/2016JC011760

Fairall, C. W., Bradley, E. F., Hare, J. E., Grachev, A. A., \& Edson, J. B. $\quad(2003$, 02). Bulk Parameterization of Air-Sea Fluxes: Updates and Verification for the COARE Algorithm. Journal of Climate, 16(4), 571-591. Retrieved from https://doi .org/10.1175/1520-0442(2003) 016<0571: BPOASF>2.0.CO;2 doi: 10.1175/1520-0442(2003)016 $\langle 0571$ :BPOASF $\rangle 2.0 . C O ; 2$

Garreau, P., Dumas, F., Louazel, S., Stegner, A., \& Le Vu, B. $\quad$ (2018). Highresolution observations and tracking of a dual-core anticyclonic eddy in the algerian basin. Journal of Geophysical Research: Oceans, 123(12), 9320-9339. Retrieved from https://agupubs.onlinelibrary.wiley.com/doi/abs/ 10.1029/2017JC013667 doi: 10.1029/2017JC013667

Gaultier, L., Ubelmann, C., \& Fu, L.-L. $\quad$ (01 Jan. 2016). The challenge of using future swot data for oceanic field reconstruction. Journal of Atmospheric and Oceanic Technology, 33(1), 119 - 126. Retrieved from https:// journals.ametsoc.org/view/journals/atot/33/1/jtech-d-15-0160_1.xml doi: 10.1175/JTECH-D-15-0160.1

Graves, L. P., McWilliams, J. C., \& Montgomery, M. T. (2006). Vortex evolution due to straining: a mechanism for dominance of strong, interior anticyclones. $\quad$ Geophysical \& Astrophysical Fluid Dynamics, 100(3), 151-183. Retrieved from https://doi.org/10.1080/03091920600792041 doi: 10.1080/03091920600792041

Ioannou, A., Stegner, A., Dumas, F., \& Le Vu, B. (2020). Three-dimensional evolution of mesoscale anticyclones in the lee of crete. Frontiers in Marine Science, 7. 1019

Ioannou, A., Stegner, A., Le Vu, B., Taupier-Letage, I., \& Speich, S. (2017). Dynamical evolution of intense ierapetra eddies on a 22 year long period. Journal of Geophysical Research: Oceans, 122(11), 9276-9298. Retrieved from https:// agupubs.onlinelibrary.wiley.com/doi/abs/10.1002/2017JC013158 doi: 10.1002/2017JC013158

Ioannou, A., Stegner, A., Tuel, A., LeVu, B., Dumas, F., \& Speich, S. (2019). Cyclostrophic corrections of aviso/duacs surface velocities and its application to mesoscale eddies in the mediterranean sea. Journal of Geophysical Research: Oceans, 124(12), 8913-8932. Retrieved from https:// agupubs.onlinelibrary.wiley.com/doi/abs/10.1029/2019JC015031 doi: 10.1029/2019JC015031

Laxenaire, R., Speich, S., Blanke, B., Chaigneau, A., Pegliasco, C., \& Stegner, A. (2018). Anticyclonic eddies connecting the western boundaries of indian and atlantic oceans. Journal of Geophysical Research: Oceans, 123(11), 76517677. Retrieved from https://agupubs.onlinelibrary.wiley.com/doi/abs/ 10.1029/2018JC014270 doi: 10.1029/2018JC014270

Laxenaire, R., Speich, S., \& Stegner, A. (2019). Evolution of the thermohaline structure of one agulhas ring reconstructed from satellite altimetry and argo floats. Journal of Geophysical Research: Oceans, 124(12), 8969-9003. Retrieved from https://agupubs .onlinelibrary.wiley.com/doi/abs/10.1029/ 2018JC014426 doi: 10.1029/2018JC014426

Lazar, A., Stegner, A., \& Heifetz, E. (2013). Inertial instability of intense stratified anticyclones. part 1. generalized stability criterion. Journal of Fluid Mechanics, 732, 457-484. doi: 10.1017/jfm.2013.412 
Le Traon, P. Y., Nadal, F., \& Ducet, N. Method of Multisatellite Altimeter Data. Oceanic Technology, 15(2), 522-534.

1998, 04)

An Improved Mapping Journal of Atmospheric and 10.1175/1520-0426(1998)015<0522:AIMMOM>2 .0.C0;2 doi: $10.1175 /$ 1520-0426(1998)015<0522:AIMMOM $>2.0 . \mathrm{CO} ; 2$

Le Vu, B., Stegner, A., \& Arsouze, T. $\quad(2018,04)$. Angular Momentum Eddy Detection and Tracking Algorithm (AMEDA) and Its Application to Coastal Eddy Formation. Journal of Atmospheric and Oceanic Technology, 35(4), 739-762. Retrieved from https://doi.org/10.1175/JTECH-D-17-0010.1 doi: 10.1175/JTECH-D-17-0010.1

Lévy, M., Franks, P. J. S., \& Smith, K. S. (2018, December). The role of submesoscale currents in structuring marine ecosystems. Nature Communications, 9(1), 4758. Retrieved from https://hal.sorbonne-universite.fr/ hal-01924116 doi: 10.1038/s41467-018-07059-3

Linden, P. F., Boubnov, B. M., \& Dalziel, S. B. (1995). Source-sink turbulence in a rotating stratified fluid. Journal of Fluid Mechanics, 298, 81-112.

Mason, E., Pascual, A., \& McWilliams, J. C. (2014). A new sea surface height-based code for oceanic mesoscale eddy tracking. Journal of Atmospheric and Oceanic Technology, 31(5), 1181-1188. Retrieved from http://dx.doi.org/10.1175/ JTECH-D-14-00019.1 doi: 10.1175/JTECH-D-14-00019.1

Mcgillicuddy, D., Robinson, A., Siegel, D., Jannasch, H., Johnson, R., Dickey, T., ... Knap, A. (1998, 07). Influence of mesoscale eddies on new production in the sargasso sea. Nature, 394, 263-266. doi: 10.1038/28367

Mkhinini, N., Coimbra, A. L. S., Stegner, A., Arsouze, T., Taupier-Letage, I., \& Béranger, K. (2014). Long-lived mesoscale eddies in the eastern mediterranean sea: Analysis of 20 years of aviso geostrophic velocities. Journal of Geophysical Research: Oceans, 119(12), 8603-8626. Retrieved from http://dx.doi.org/10.1002/2014JC010176 doi: 10.1002/2014JC010176

Nencioli, F., Dall'Olmo, G., \& Quartly, G. D. (2018). Agulhas ring transport efficiency from combined satellite altimetry and argo profiles. Journal of Geophysical Research: Oceans, 123(8), 5874-5888. Retrieved from https://agupubs .onlinelibrary.wiley.com/doi/abs/10.1029/2018JC013909 doi: 10.1029/ 2018JC013909

Nencioli, F., Dong, C., Dickey, T., Washburn, L., \& McWilliams, J. C.

(2010). A vector geometry-based eddy detection algorithm and its application to a high-resolution numerical model product and high-frequency radar surface velocities in the southern california bight. Journal of Atmospheric and Oceanic Technology, 27(3), 564-579.

Penven, P., Halo, I., Pous, S., \& Marié, L. (2014). Cyclogeostrophic balance in the mozambique channel. Journal of Geophysical Research: Oceans, 119(2), 10541067.

Perret, G., Dubos, T., \& Stegner, A. (2011, February). How large-scale and cyclogeostrophic barotropic instabilities favor the formation of anticyclonic vortices in the ocean. Journal of Physical Oceanography, 41(2), 303-328. Retrieved from https://hal-ensta-paris.archives-ouvertes.fr//hal-00838872 doi: $10.1175 / 2010$ jpo4362.1

Perret, G., Stegner, A., Farge, M., \& Pichon, T. (2006). Cyclone-anticyclone asymmetry of large-scale wakes in the laboratory. Physics of Fluids, 18(3), 036603. Retrieved from https://doi.org/10.1063/1.2179387 doi: 10.1063/1.2179387

Polvani, L. M., McWilliams, J. C., Spall, M. A., \& Ford, R. (1994). The coherent structures of shallow-water turbulence: Deformation-radius effects, cyclone/anticyclone asymmetry and gravity-wave generation. Chaos: An Interdisciplinary Journal of Nonlinear Science, 4(2), 177-186. Retrieved from https://doi.org/10.1063/1.166002 doi: 10.1063/1.166002 
Prants, S., Uleysky, M., \& Budyansky, M. structures in the ocean favorable for fishery. 1269-1272. doi: 10.1134/S1028334X12110062

Puillat, I., Taupier-Letage, I., \& Millot, C. (2002). Algerian eddies lifetime can near 3 years. Journal of Marine Systems, 31(4), 245-259.

Pujol, I., \& Larnicol, G. (2005, 12). Mediterranean sea eddy kinetic energy variability from 11 years of altimetric data. Journal of Marine Systems - J MARINE SYST, 58, 121-142. doi: 10.1016/j.jmarsys.2005.07.005

Shchepetkin, A. F., \& McWilliams, J. C. (2005). The regional oceanic modeling system (roms): a split-explicit, free-surface, topography-followingcoordinate oceanic model. Ocean Modelling, 9(4), 347-404. Retrieved from http://www.sciencedirect.com/science/article/pii/S1463500304000484 doi: http://dx.doi.org/10.1016/j.ocemod.2004.08.002

Stegner, A. (2014). Oceanic island wake flows in the laboratory. In T. von Larcher \& P. D. W. J. W. . Sons (Eds.), Modeling atmospheric and oceanic flows (p. 265-276). American Geophysical Union (AGU). Retrieved from https:// agupubs .onlinelibrary.wiley.com/doi/abs/10.1002/9781118856024.ch14 doi: $10.1002 / 9781118856024 . \operatorname{ch} 14$

Stegner, A., \& Dritschel, D. G. $\quad(2000,10)$ A Numerical Investigation of the Stability of Isolated Shallow Water Vortices. Journal of Physical Oceanography, 30(10), 2562-2573. Retrieved from https://doi.org/10.1175/ 1520-0485 (2000) 030<2562: ANIOTS>2 .0. C0;2 doi: 10.1175/1520-0485(2000) 030<2562:ANIOTS $>2.0 . \mathrm{CO} ; 2$

Stegner, A., Le Vu, B., Pegliasco, C., Moschos, E., \& Faugère, Y. (2019). 3d structure of long-lived eddies in the mediterranean sea : the dyned-atlas database (Vol. 42) [Article]. Retrieved from http://ciesm.org/online/archives/ abstracts/pdf/42/CIESM_Congress_2019_Cascais_article_0074.pdf

Taburet, G., Sanchez-Roman, A., Ballarotta, M., Pujol, M.-I., Legeais, J.-F., Fournier, F., ... Dibarboure, G. (2019). Duacs dt2018: 25 years of reprocessed sea level altimetry products. Ocean Science, 15(5), 1207-1224. Retrieved from https://os.copernicus.org/articles/15/1207/2019/ doi: 10.5194/os-15-1207-2019

Watson, J. R., Fuller, E. C., Castruccio, F. S., \& Samhouri, J. F. (2018). Fishermen follow fine-scale physical ocean features for finance. Frontiers in Marine Science, 5, 46. Retrieved from https://www.frontiersin.org/article/10 .3389/fmars.2018.00046 doi: 10.3389/fmars.2018.00046

Yim, E., Stegner, A., \& Billant, P. $\quad(2019,03)$. Stability Criterion for the Centrifugal Instability of Surface Intensified Anticyclones. Journal of Physical Oceanography, $49(3), 827-849$. Retrieved from https://doi.org/10.1175/JPO-D-18 -0088. 1 doi: 10.1175/JPO-D-18-0088.1 

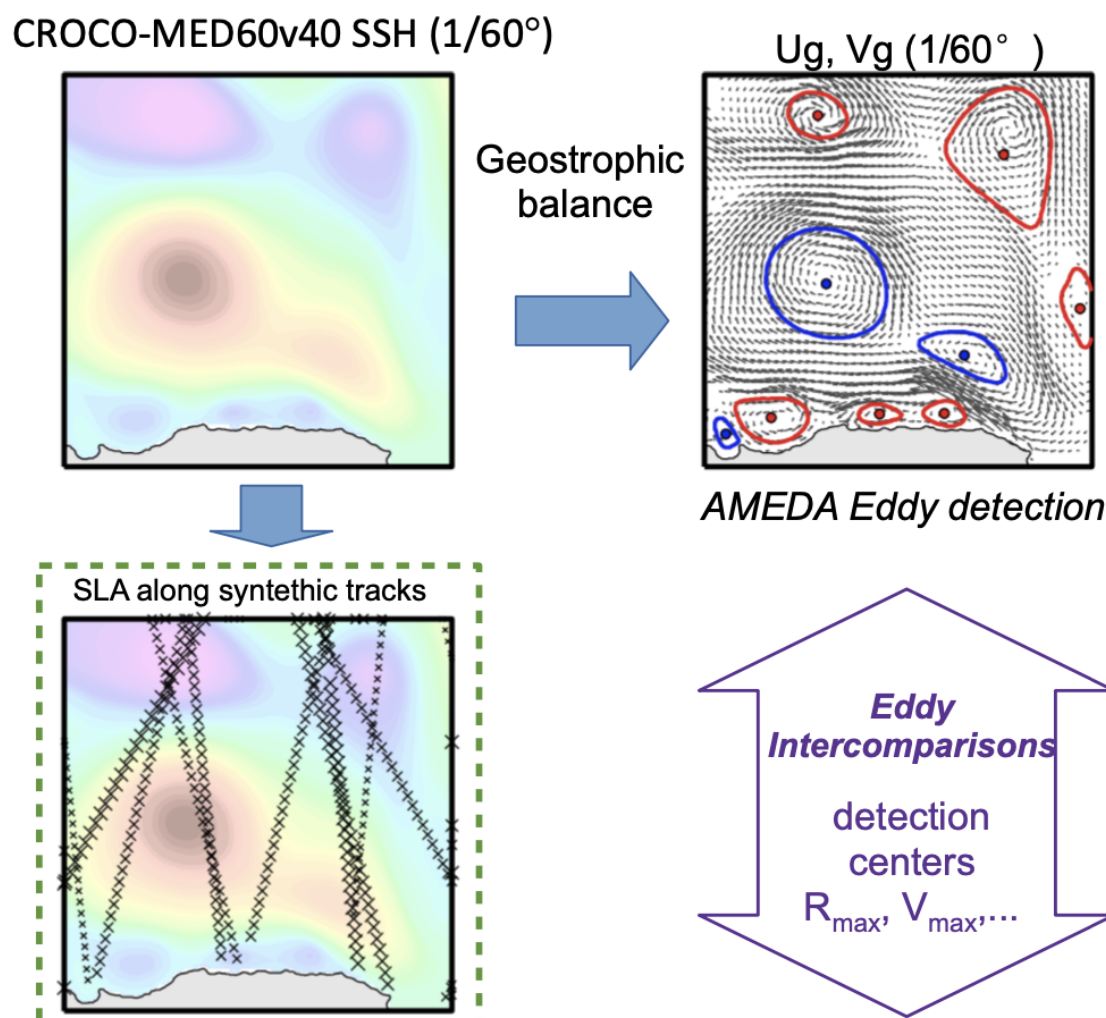

AMEDA Eddy detection

Optimal interpolation (CLS)
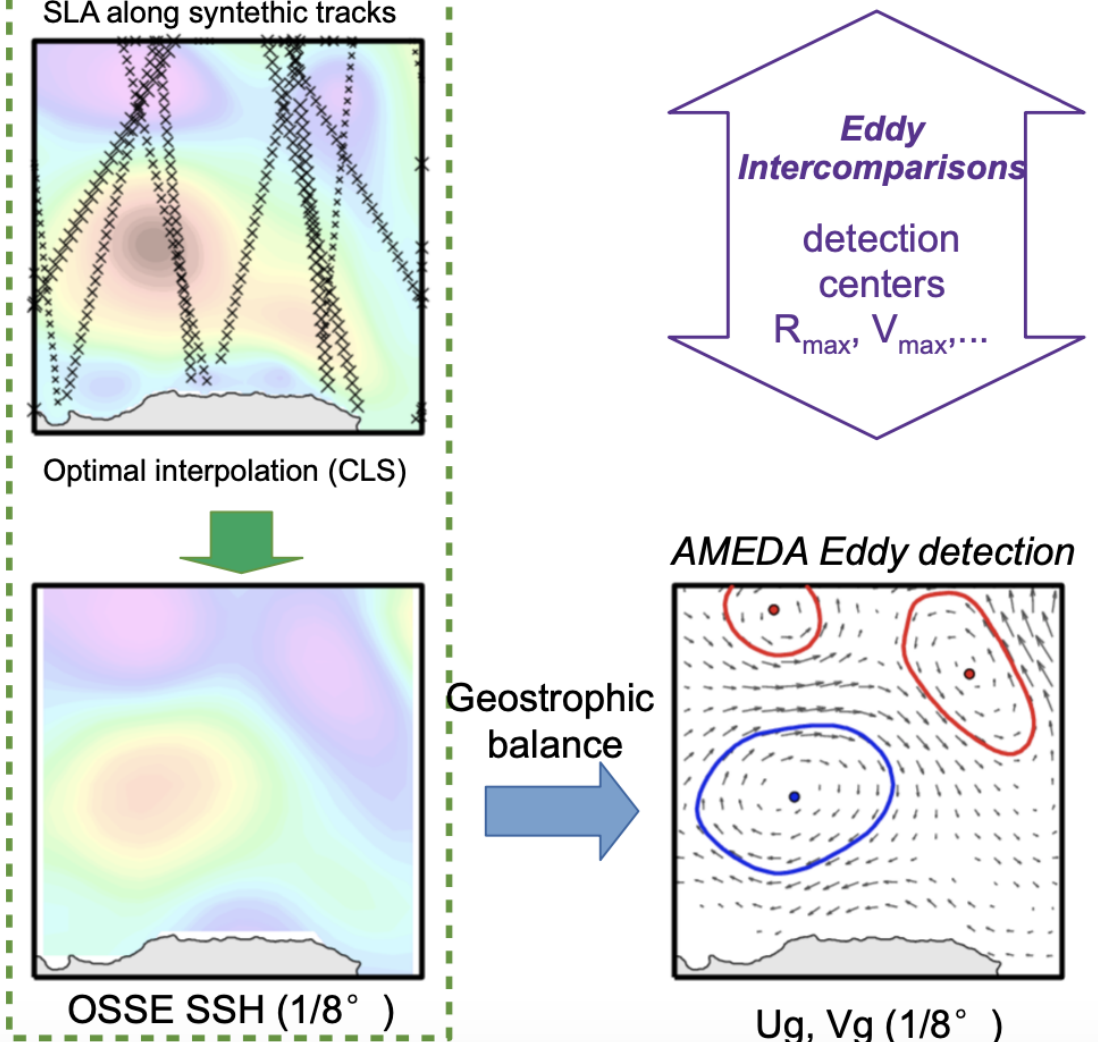

AMEDA Eddy detection

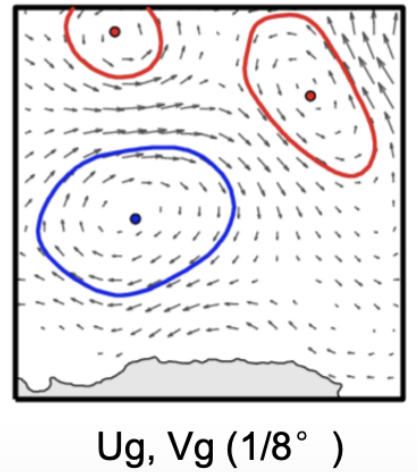

Figure 1. Principle of the Observing System Simulation Experiment (OSSE) and methodology followed 


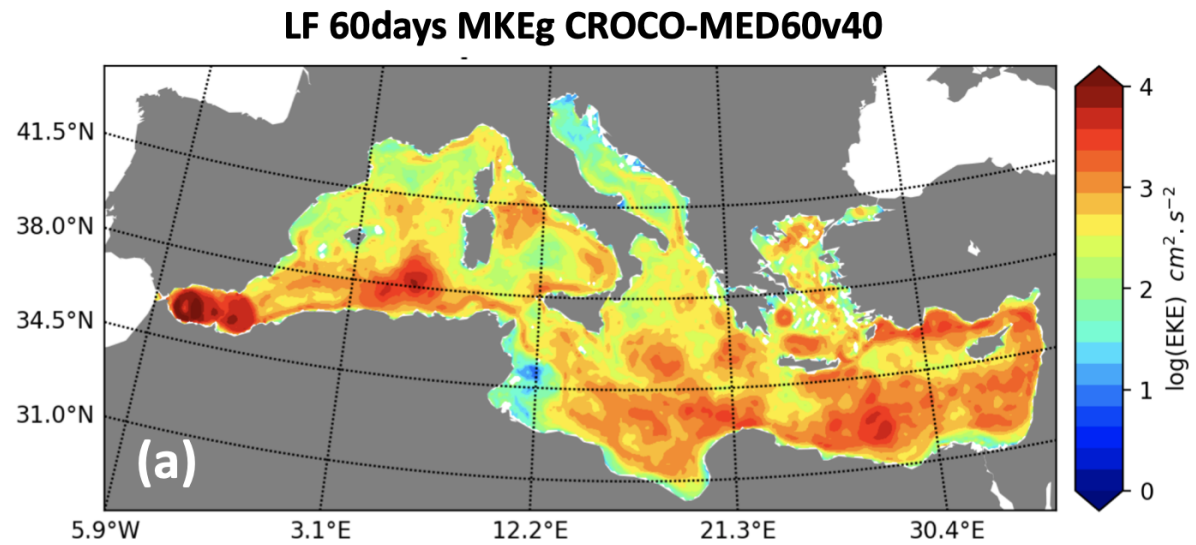

LF 60days MKEg AVISO/DUACS

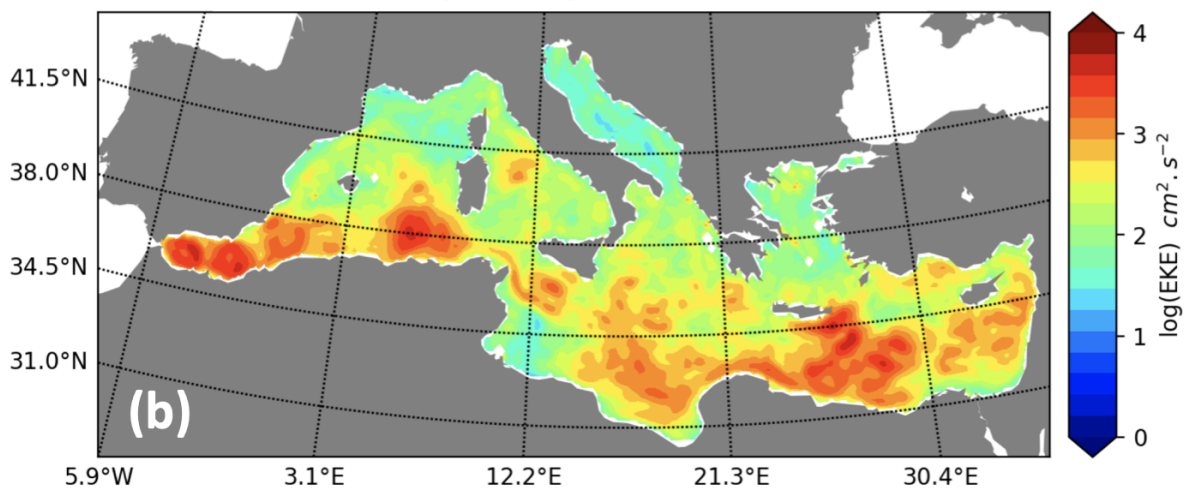

Figure 2. Mean kinetic energy (MKEg) of the CROCO-MED60v40 (a) and the AVISO/CMEMS altimetry products (b) computed from the low-frequency (60 days cut-off) geostrophic velocity field 


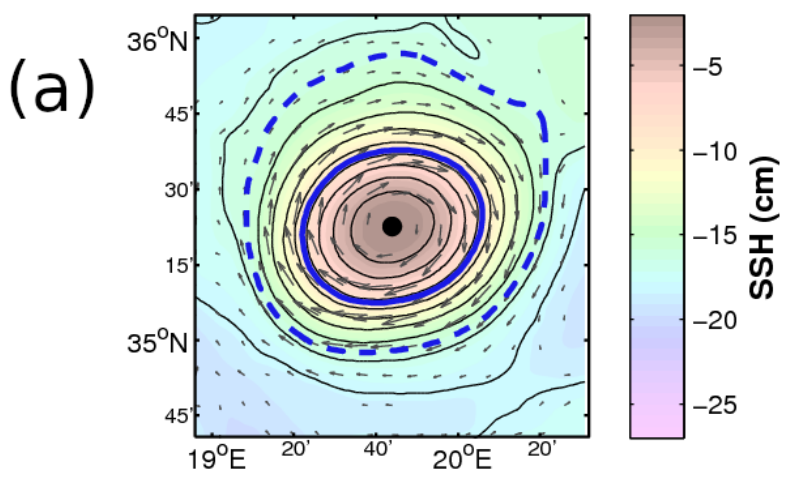

(b)

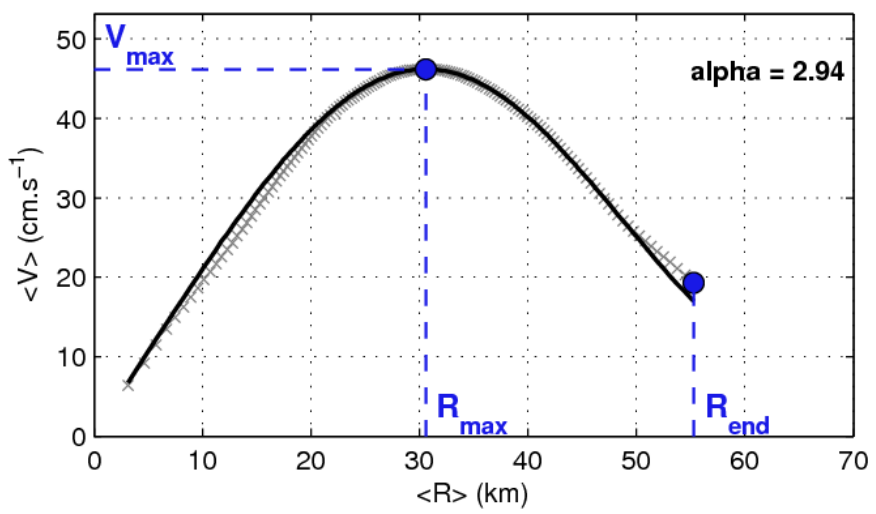

(c)

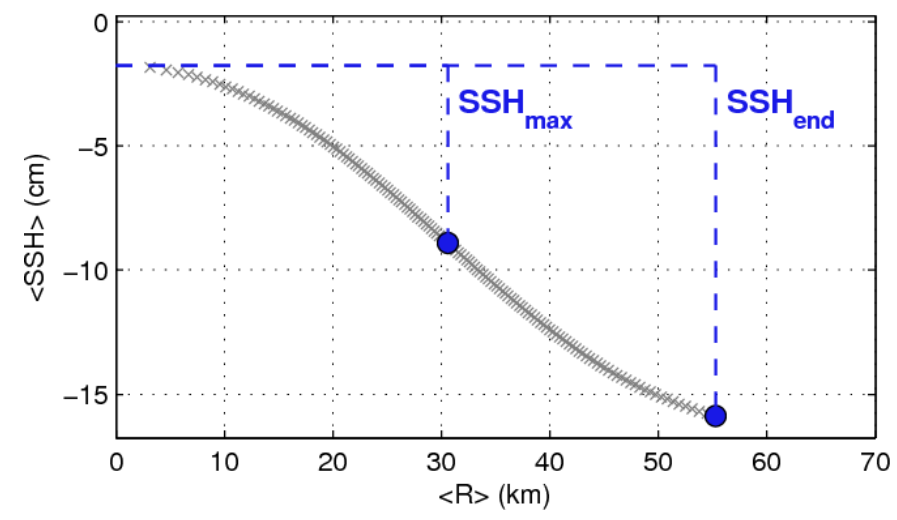

Figure 3. The first panel (a) shows the characteristic contour (blue solid line) and the last closed contour (blue dashed line) calculated by the AMEDA algorithm for an anticyclone. The background colors correspond to the SSH fields and the black vectors to the surface velocity components. The mean velocity profile $\langle V\rangle=F(\langle R\rangle)$ deduced from the streamlines analysis is plotted with grey crosses the central panel (b). The black curve correspond to the fit with the generic velocity profile eqn.(3) with a steepness parameter $\alpha=2.94$. The lower panel (c) depicts the mean radial profile of the eddy amplitude from the eddy center to the last closed contour (i.e. $\left.R_{e n d}\right)$. 



Figure 4. Histogram of the radius $R_{\max }$ (a) and the amplitudes $\eta_{\text {eddy }}$ (b) of all the detected eddies on the CROCO-MED60v40-15-16 (black curve), the OSSE-DT (pink solid curve) and the OSSE-NRT (pink dashed curve). The corresponding histograms of the DYNED-Atlas data base (for 2015-2016) is plotted with a green curve.
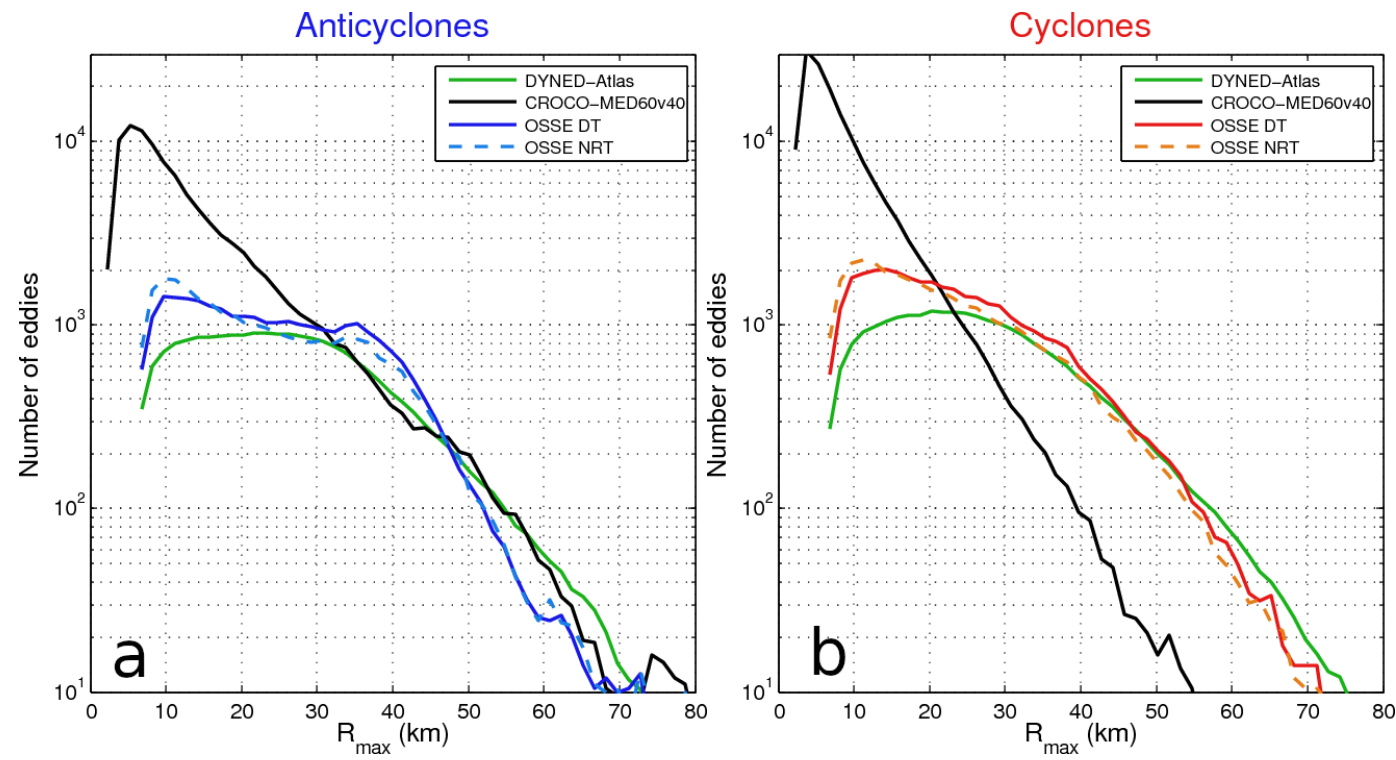

Figure 5. Histogram of the radius $R_{\max }$ of the detected anticyclones (a) and cyclones (b). The curves corresponding to the CROCO-MED60v40-15-16 and the DYNED-Atlas data base are plotted in black and green respectively, while the OSSE-DT (OSSE-NRT) are plotted with solid (dashed) blue or red lines. 

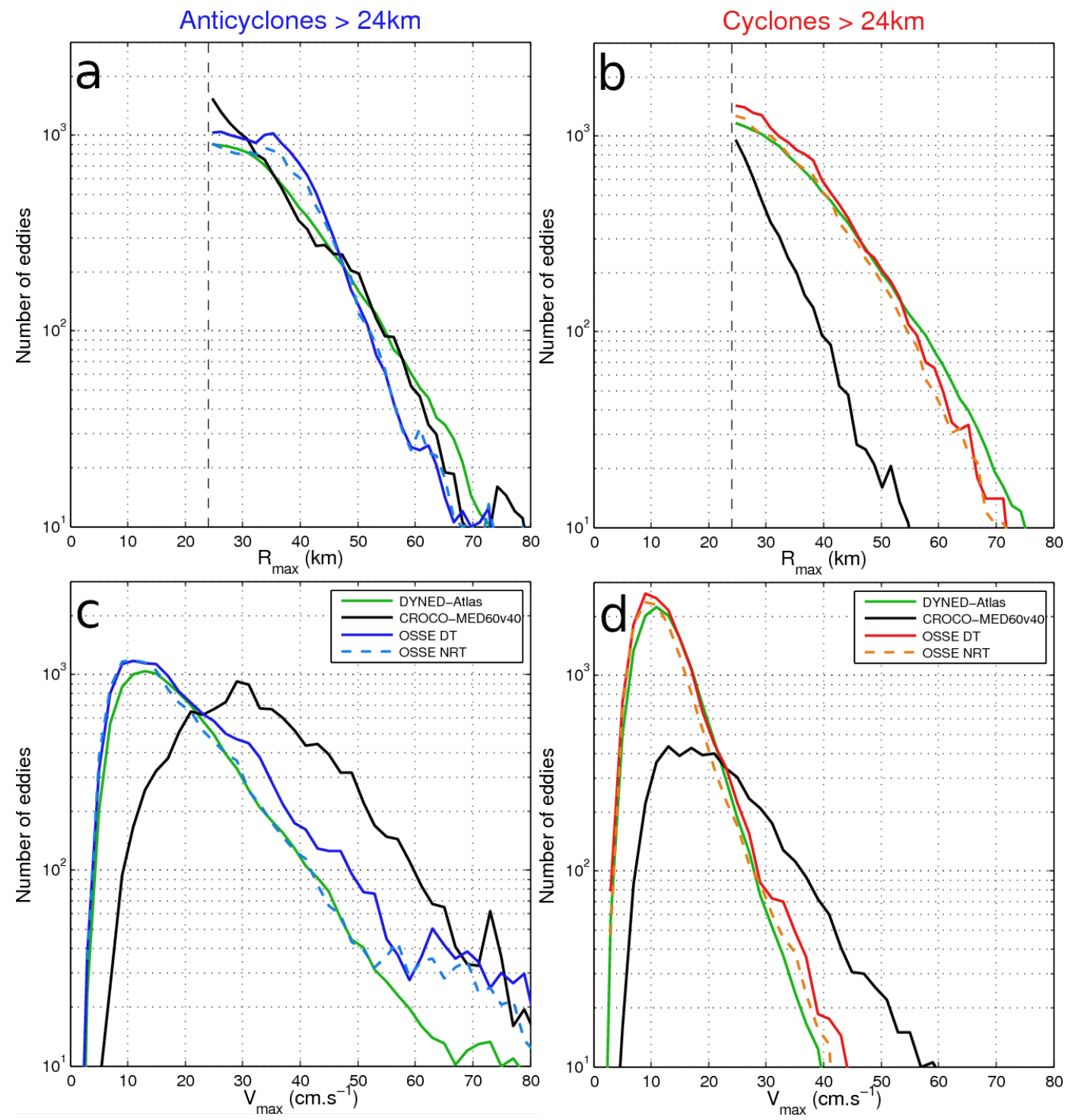

Figure 6. Histogram of the characteristic radius $R_{\max }$ and the intensity $V_{\max }$ of large mesoscale eddies (with $R_{\max }>24 k m$ ) of both sign anticyclones (a,c) and cyclones (b,d). The curves colors are identical to the previous Figure 4 

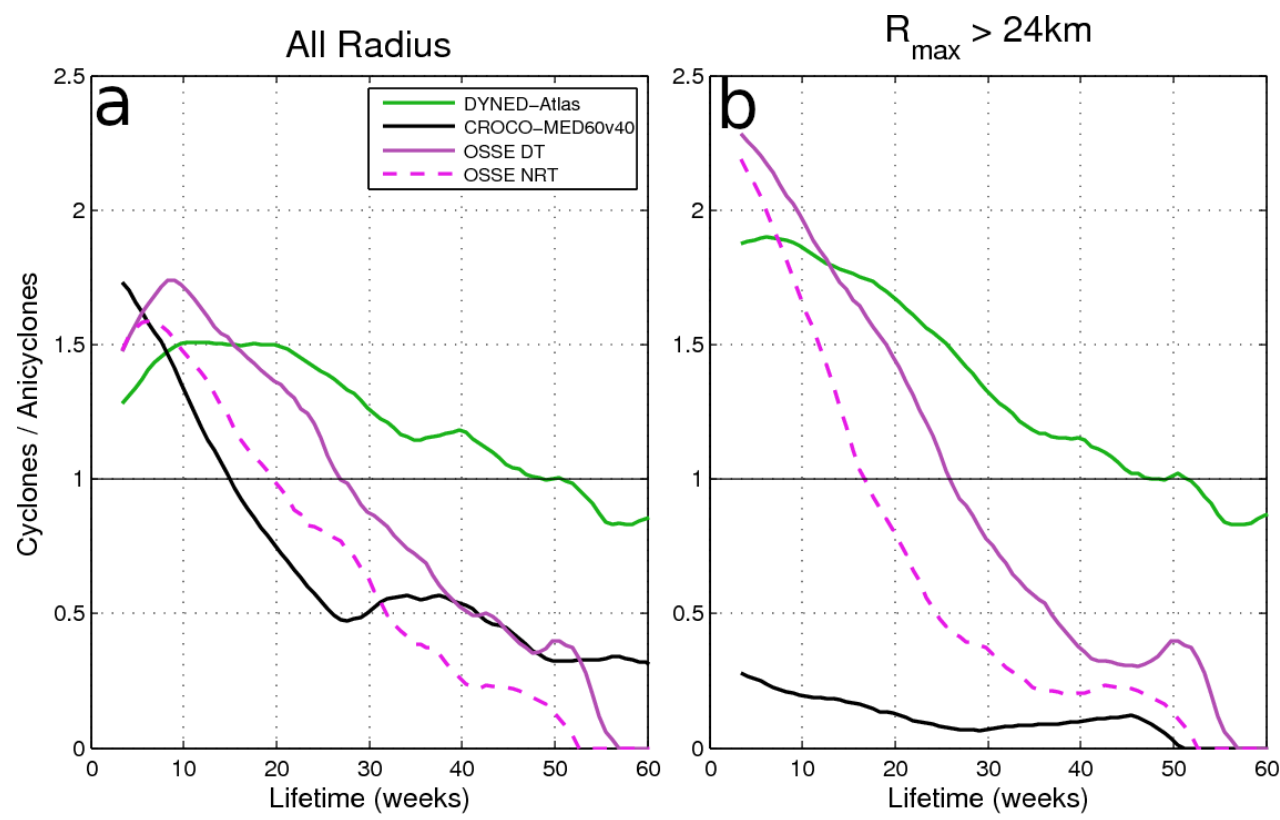

Figure 7. The cumulative histograms of the ratio cyclones/anticyclones for eddies with lifetimes greater than or equal to each particular value along the abscissa. The left panel (a) corresponds to the unfiltered histograms where all the detected eddies are considered, while the histograms of large mesoscale eddies (with $R_{\max }>24 \mathrm{~km}$ ) are plotted in the right panel (b). The ratio cyclones/anticyclones for CROCO-MED60v40-15-16, the OSSE-DT, the OSSE-NRT and the DYNED-Atlas data base is plotted with black solid, pink solid, pink dashed and green curves respectively. 
REFERENCE
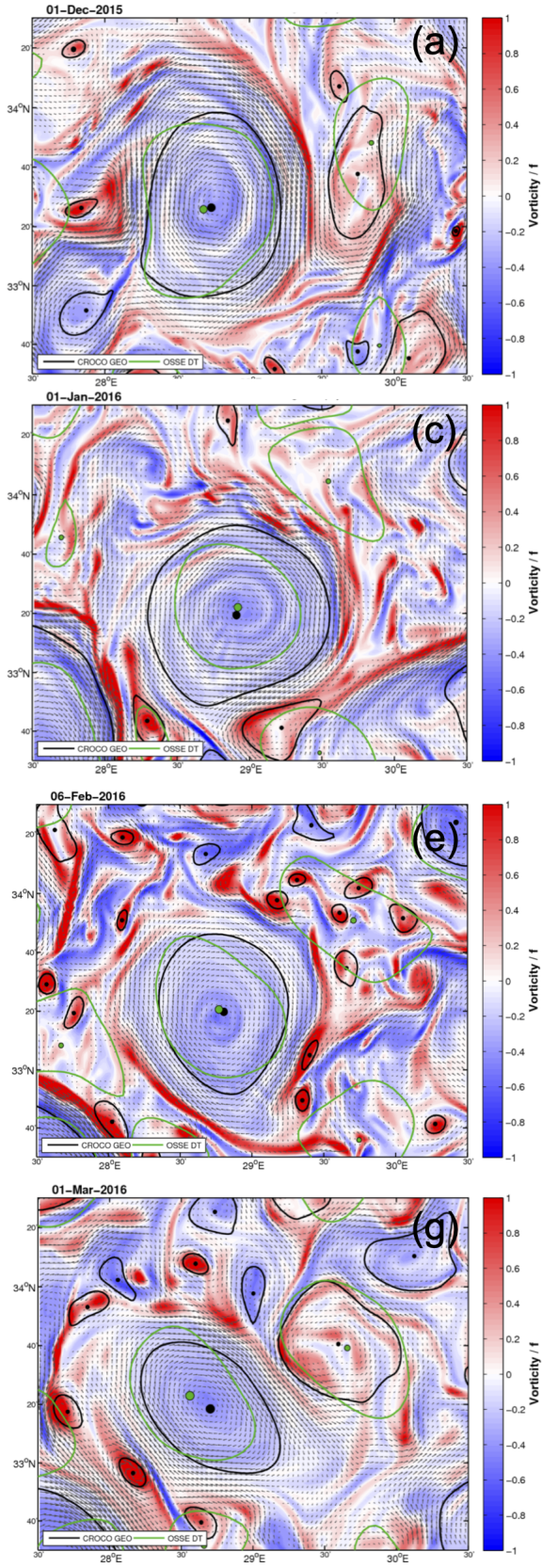

OSSE
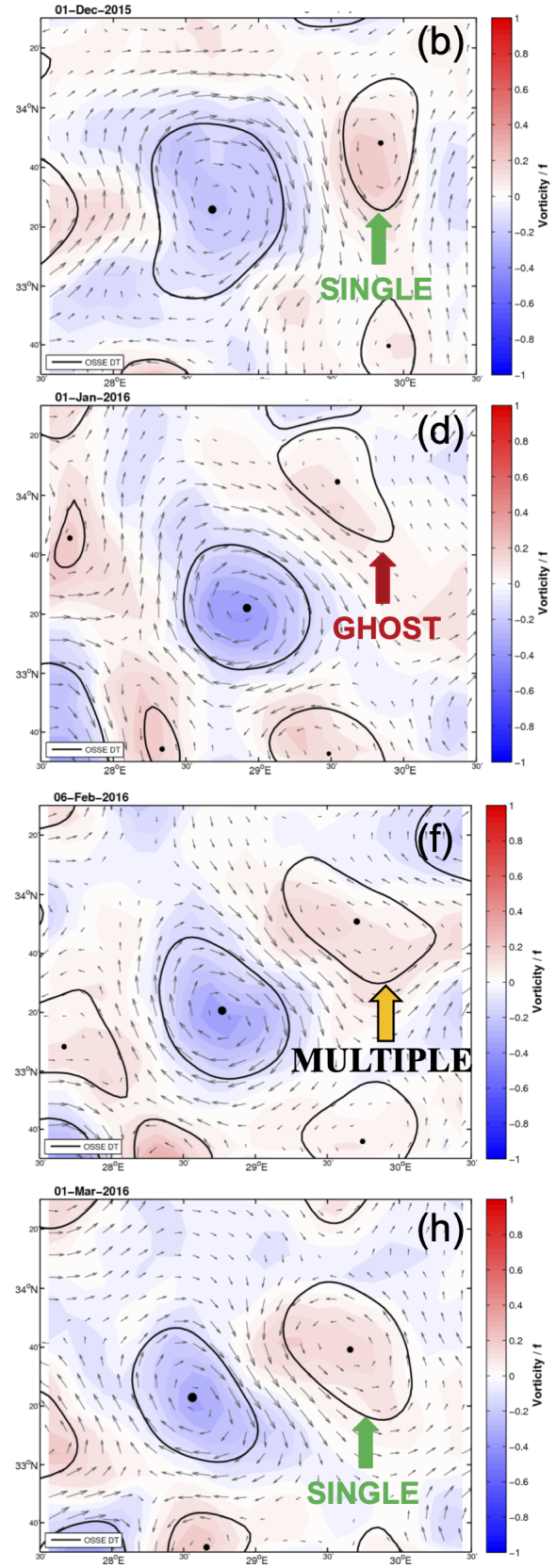

Figure 8. The temporal evolution of a large scale anticyclone and several cyclones, in its surrounding, is displayed from top to bottom: the 15 December 2015 (a,b), the 1 January 2016 $(\mathrm{c}, \mathrm{d})$, the 6 February 2016 (e,f) and the first of March $2016(\mathrm{~g}, \mathrm{~h})$ in the center of the Levantine basin. Theses snapshots of the surface geostrophic velocity (black arrows) and the relative vorticity (background color) are presented side by side for the CROCO-MED60v40-15-16 (a,c,e,g) and the OSSE-DT $(b, d, f, h)$. The characteristic contours of all the eddies detected by the AMEDA algorithm on each field are plotted in black. The contours of the eddies detected on the OSSE-DT are superimposed on the reference field (i.e. the CROCO-MED60v40-15-16) with green contours for better comparison. 

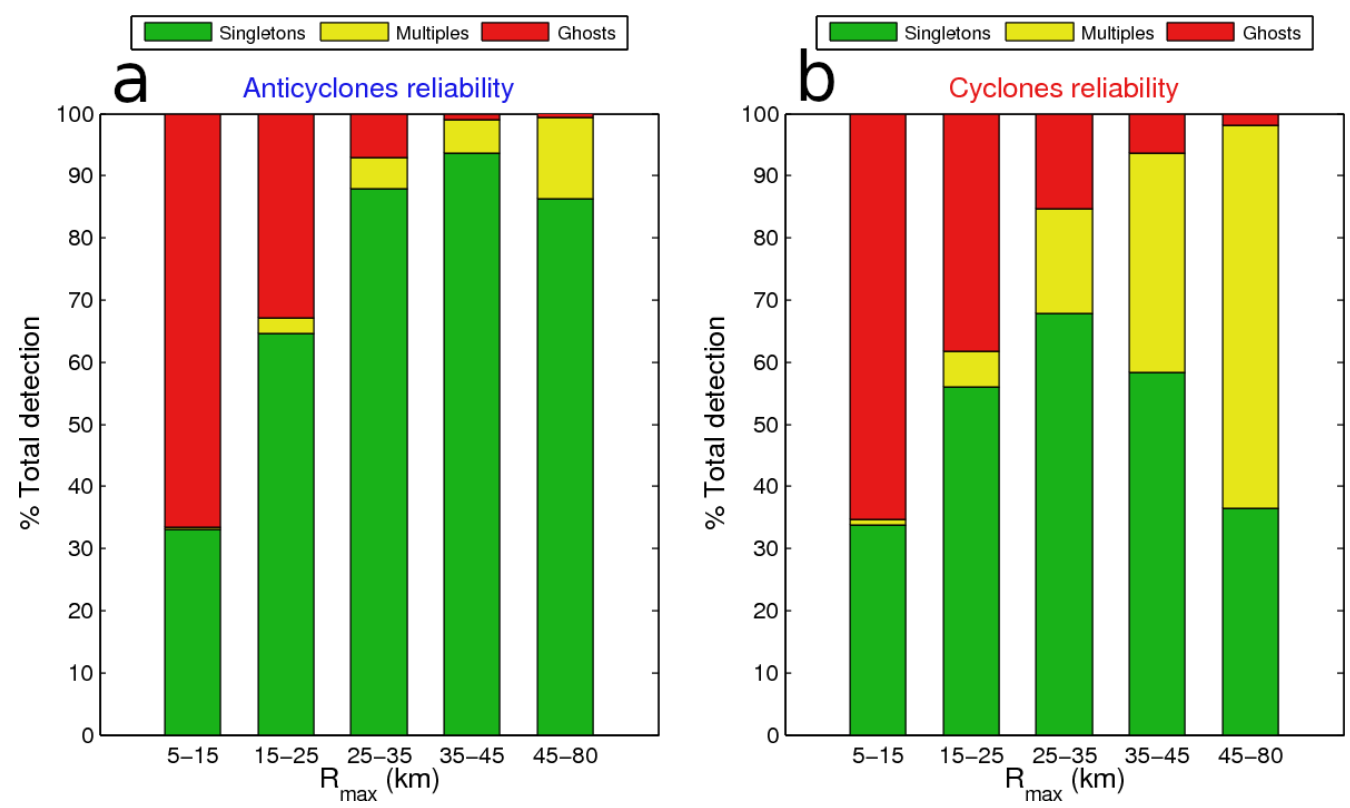

Figure 9. The proportion of ghost (red), multiple (yellow) and single (green) anticyclones (a) and cyclones (b) detected on the OSSE-DT are plotted as a function of their size (i.e. $R_{\max }$ ). 

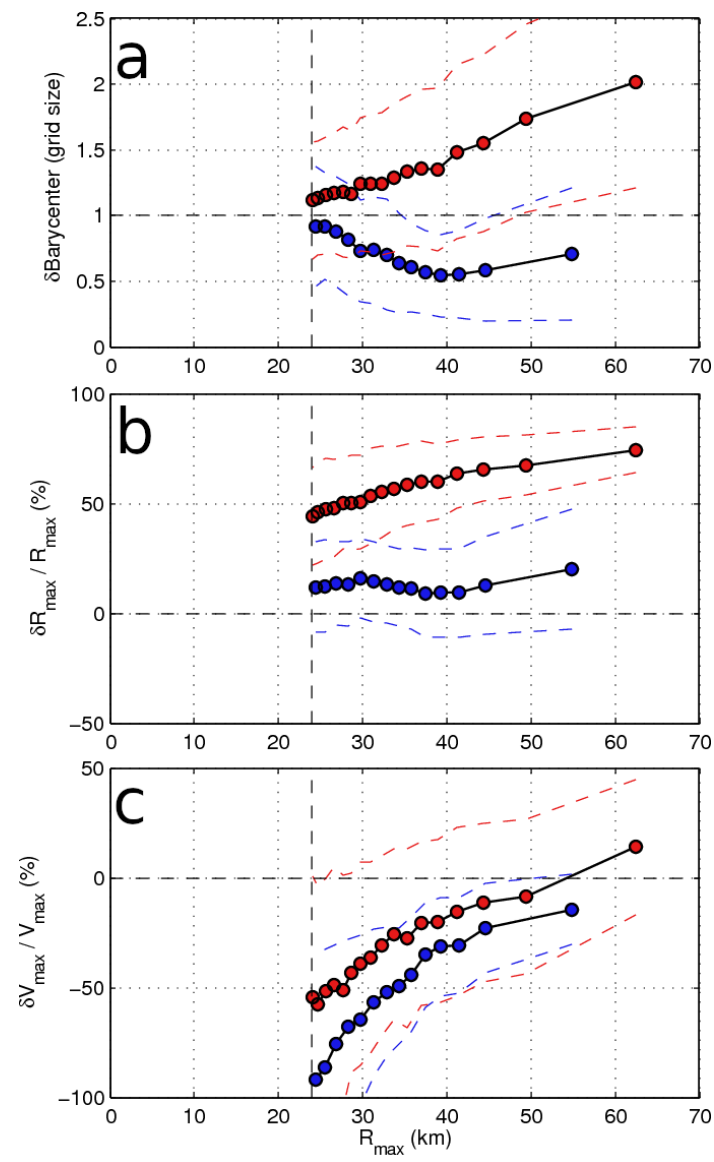

Figure 10. Normalized errors of the barycenter position (a), the size (b) and the intensity (c) of large mesoscale eddies detected on the OSSE-DT. The mean error values and their interquartile range (between $25^{t h}$ and $75^{t h}$ percentile) are plotted, as function of the eddy radius $R_{\max }$, with circle dots and dashed lines respectively. Cyclonic and anticyclonic eddies are plotted separately with red and blue dots. 

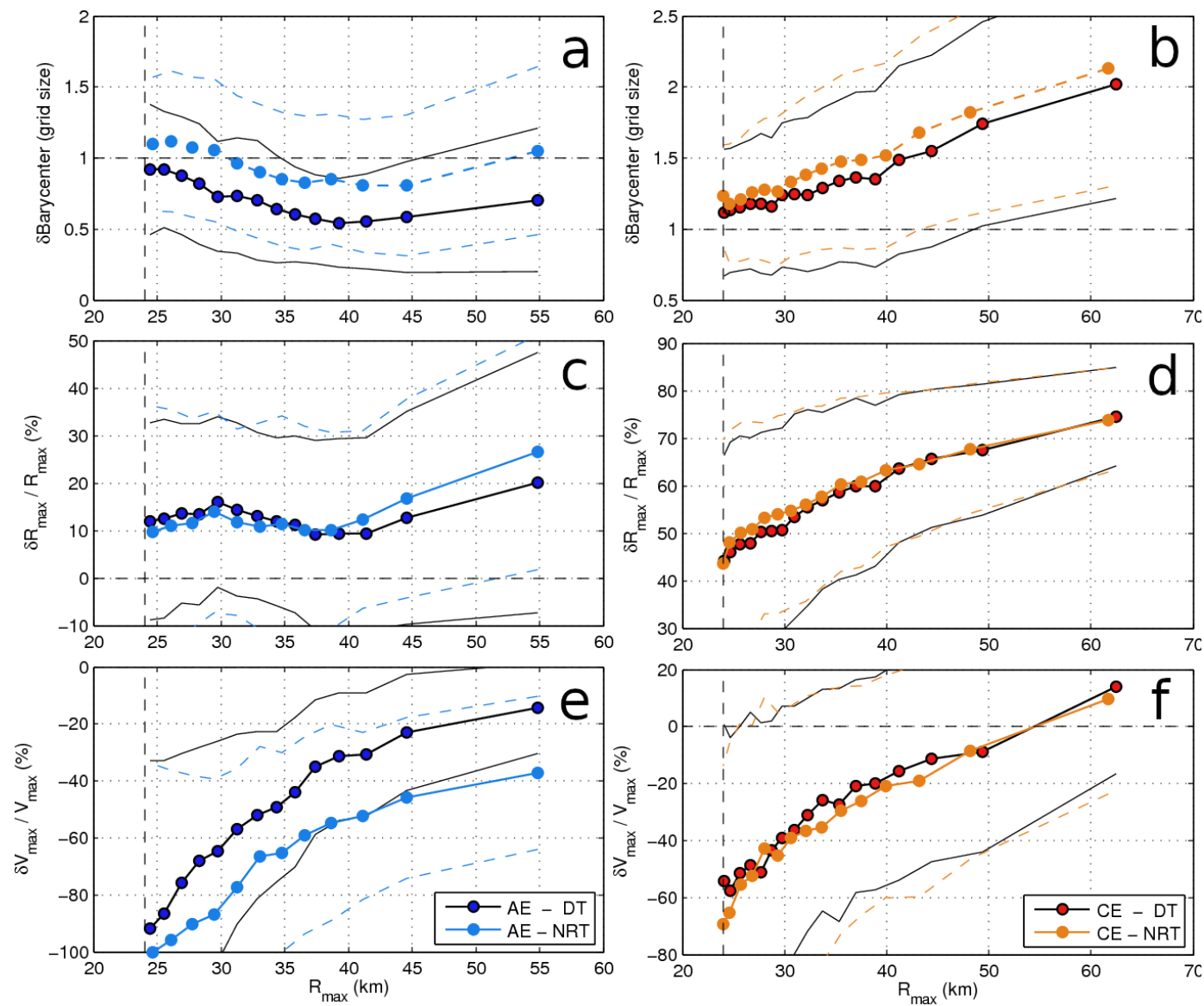

Figure 11. Comparisons between the normalized errors of large mesoscale eddies detected on the OSSE-DT and the OSSE-NRT. The analysis corresponding to anticyclonic eddies are plotted on the left panels $(\mathrm{a}, \mathrm{c}, \mathrm{e})$ while cyclonic ones are on the right panels $(\mathrm{b}, \mathrm{d}, \mathrm{f})$. 